\title{
Synthesis and Properties of Dichlorovinyl Derivatives of Tetrazoles
}

\author{
L. V. Myznikov ${ }^{a, *}$, S. V. Vorona ${ }^{a}$, A. S. Lyakhov ${ }^{b}$, L. S. Ivashkevich ${ }^{b}$, and Yu. E. Zevatskii ${ }^{c}$ \\ ${ }^{a}$ Institute of Experimental Medicine, St. Petersburg, 197376 Russia \\ ${ }^{b}$ Department of the Belarusian State University "Research Institute of Physical and Chemical Problems," Minsk, 220006 Belarus \\ ${ }^{c}$ St. Petersburg State Institute of Technology (Technical University), St. Petersburg, 190013 Russia \\ *e-mail:myznikov_lv@mail.ru
}

Received July 1, 2021; revised July 1, 2021; accepted July 14, 2021

\begin{abstract}
Substituted $1 H$-tetrazole-5-thiols and 5-substituted $1 H$-tetrazoles easily reacted with trichloroethylene to form the corresponding $S$ - and $\mathrm{N}$-dichlorovinyl derivatives, respectively. In the case of 5 -substituted $1 \mathrm{H}$ tetrazoles, the reaction led to a mixture of 1- and 2-dichlorovinyltetrazoles. 5-Substituted-2-dichlorovinyltetrazoles are characterized by low thermal stability, but easily enter into the polymerization reaction.
\end{abstract}

Keywords: 1 -substituted $1 H$-tetrazole-5-thiols, 5 -substituted $1 H$-tetrazoles, trichlorethylene, dichlorovinyl derivatives

DOI: $10.1134 / \mathrm{S} 1070363221090061$

Vinyl derivatives of (thio)tetrazoles attract attention as starting materials for the synthesis of high molecular weight compounds, biologically active substances, and functional materials [1-5]. Vinyltetrazole-based polymers are components of promising energy-intensive materials, gas separation membranes, nonwoven filter materials for medicine, and other composite materials $[1,2]$.

To date, the problem of synthesizing unsubstituted vinyltetrazoles has been successfully solved. Although the known methods for the preparation of these compounds are multistage, they make it possible to obtain the corresponding vinyl derivatives in good yields $[2,6]$. In contrast, almost all known methods for the preparation of substituted vinyltetrazoles can be reduced to two main groups: the functionalization of previously obtained vinyltetrazoles using metal-catalyzed cross-coupling [7] and the addition reaction of thiotetrazoles to the activated triple bond $\mathrm{C} \equiv \mathrm{C}[8]$. Both of these reaction groups make it possible to obtain only a limited range of the products, with aromatic substituents in the first case and, usually, with electron-withdrawing carboxyl or keto groups in the second. As for the vinyl chloride derivatives of tetrazoles, no method for their preparation has been proposed to date.
One of the simple ways to introduce a chlorovinyl group could be nucleophilic substitution of halogen at the double bond; however, such reactions are hindered due to the low reactivity of substituted vinyl chlorides. At the same time, with an increase in the number of chlorine atoms, the reactivity increases significantly, and trichlorethylene can already react with strong nucleophiles to form halogen substitution products [9]. Since 1-substituted tetrazole-5-thiols and 5-substituted tetrazoles are rather strong nucleophiles, it can be expected that their reaction with trichlorethylene will lead to dichlorovinylthio- and dichlorovinyltetrazoles. Taking into account the prospects for the use of these compounds, the study of this reaction is an urgent task.

1-Substituted $1 H$-tetrazole-5-thiols were found to react with trichlorethylene at $80-90^{\circ} \mathrm{C}$ in the presence of $\mathrm{K}_{2} \mathrm{CO}_{3}$ in DMF to form dichlorovinyl derivatives in good yields (Scheme 1). It should be noted that we have previously shown that 1 -substituted $1 H$-tetrazole-5-thiols easily enter into the copper-catalyzed cross-coupling reaction with aryl halides [10], however, in the case of trichlorothylene, the addition of copper compounds did not have any effect on the reaction course. 
Scheme 1.

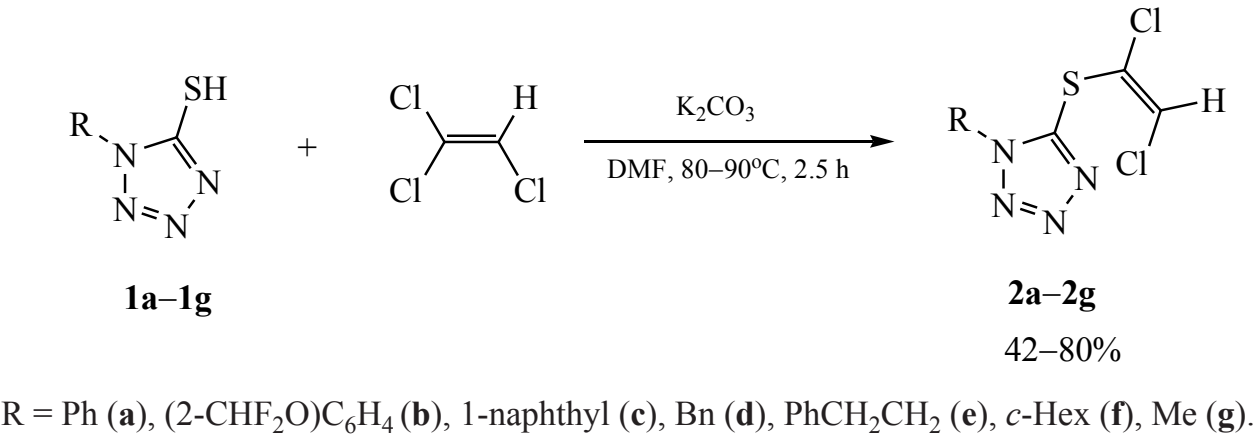

The synthesized 1-substituted dichlorovinylthiotetrazoles are stable compounds. For compound $\mathbf{2 c}(\mathrm{R}=$ 1-naphthyl), we managed to grow single crystals suitable for X-ray diffraction analysis. The crystallographic data (Table 1, Fig. 1) confirmed the expected structure of the synthesized compound. It crystallizes in the monoclinic space group $C 2 / c$, with one molecule in an asymmetric cell and eight molecules in a unit cell. The chlorine atoms of the vinyl fragment are in the trans-position. The tetrazole ring is significantly unfolded relative to the naphthyl substituent, with a dihedral angle between the root-mean-square planes of these fragments of $86.58(3)^{\circ}$. Although there are no hydrogen bonds in the crystal structure of compound $\mathbf{2 c}$, it is stabilized by $\pi-\pi-$ stacking interactions with the participation of $\pi$-systems of naphthyl fragments of neighboring molecules.

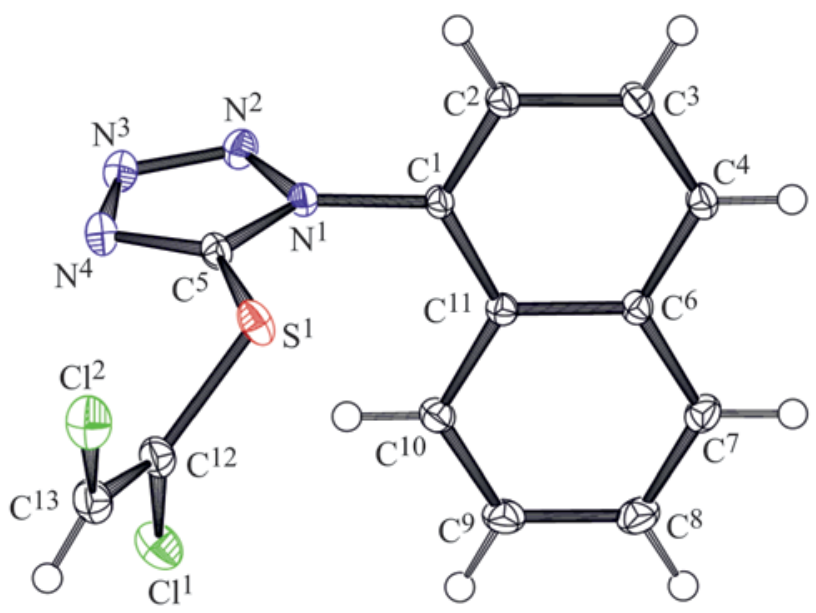

Fig. 1.General view of compound $2 \mathbf{c}$ in crystal (CCDC 2090214).
We found that the reaction of 5-phenyl-1H-tetrazole with trichlorethylene in DMF in the presence of $\mathrm{K}_{2} \mathrm{CO}_{3}$ at a temperature below $80-90^{\circ} \mathrm{C}$ gives rise to 1-dichlorovinyl-5-phenyltetrazoles with a yield of $25-35 \%$, as well as a significant amount of resinification products. This reaction outcome was found to be due to the fact that 2-dichlorovinitetrazoles have low thermal stability and decompose upon heating. Indeed, when the reaction is carried out in DMSO in the presence of $\mathrm{KOH}$, the temperature can be lowered to $40^{\circ} \mathrm{C}$. As a result, a mixture of 1- and 2-dichlorovinyltetrazoles is formed within $1-2 \mathrm{~h}$ with a total yield of $43-74 \%$. Under these conditions, we obtained a series of 1 - and 2-dichlorovinyltetrazoles with various substituents at position 5 of the tetrazole ring (Scheme 2). The ratio of 1 - and 2- isomers varies from $1.3: 1$ to $1: 2$, respectively.

In contrast to the corresponding 1-isomers and dichlorovinylthiotetrazoles, 2-dichlorovinyltetrazoles isolated in pure form have low stability and decompose when stored at room temperature, but can be stored for a long time at $-18^{\circ} \mathrm{C}$. The addition of radical initiators causes rapid polymerization of 5-substituted 2-dichlorovinyltetrazoles, which makes these compounds promising monomers for the preparation of functional materials.

In summary, trichlorethylene is a convenient andavailable substrate for the preparation of dichlorovinylthiotetrazoles, 1- and 2-dichlorovinyltetrazoles. 2-Dichlorovinyltetrazoles can be used as starting compounds for the synthesis of high molecular weight compounds.

\section{EXPERIMENTAL}

${ }^{1} \mathrm{H}$ and ${ }^{13} \mathrm{C}$ NMR spectra were recorded on a Bruker Avance III HD 400 NanoBay spectrometer (400 and $100 \mathrm{MHz}$, respectively) in $\mathrm{CDCl}_{3}$ solution, the internal 
standard was the residual signals of the solvent. IR spectra were recorded on a Shimadzu FTIR-8400S spectrometer from $\mathrm{KBr}$ pellets. Elemental analysis was performed on a LECO CHNS-932 analyzer. The purity and individuality of the obtained compounds were monitored by TLC on Merck Silicagel UV-254 plates. Melting points were determined on a Kofler table. All starting materials and solvents were of reagent or analytical grade.

Single crystal X-ray diffraction analysis of compound 2c was performed on a SMART Apex II X-ray diffractometer (Bruker AXS GmbH, Germany) using $\mathrm{MoK}_{\alpha}$ radiation (graphite monochromator). The structure was solved by direct methods using the SIR2014 program [11] and refined using $F^{2}$ by full-matrix least squares in the anisotropic approximation for non-hydrogen atoms using the SHELXL-2014 software package [12]. The positions of hydrogen atoms were calculated geometrically and refined within the framework of the rider model with $U_{\text {iso }}(\mathrm{H})=1.2 U_{\text {eq }}(\mathrm{C})$. Molecular graphics were performed using the PLATON software [13]. The obtained crystallographic data were deposited with the Cambridge Crystallographic Data Center (CCDC 2090214).

(E)-5-[(1,2-Dichlorovinyl)thio]-1-phenyl-1Htetrazole (2a). To a mixture of $3.8 \mathrm{~g}(21.3 \mathrm{mmol})$ of 1-phenyl- $1 H$-tetrazole-5-thiol and $8.81 \mathrm{~g}(63.9 \mathrm{mmol})$ of $\mathrm{K}_{2} \mathrm{CO}_{3}$ in $6 \mathrm{~mL}$ of DMF was added $8.40 \mathrm{~g}(63.9 \mathrm{mmol})$ of trichlorethylene. The reaction mixture was stirred for $2.5 \mathrm{~h}$ at $80-90^{\circ} \mathrm{C}$, then poured into $50 \mathrm{~mL}$ of cold water and extracted with chloroform $(3 \times 30 \mathrm{~mL})$. The organic layer was washed with water $(3 \times 30 \mathrm{~mL})$ and saturated $\mathrm{NaCl}$ solution, then dried with anhydrous $\mathrm{Na}_{2} \mathrm{SO}_{4}$. The solvent was removed in vacuum. Yield $4.37 \mathrm{~g}(75 \%)$, yellow crystals, $\mathrm{mp} 87-89^{\circ} \mathrm{C}(\mathrm{EtOH})$. IR spectrum, $v$, $\mathrm{cm}^{-1}(\mathrm{KBr})$ : $3109 \mathrm{~m}(\mathrm{CH}), 3031 \mathrm{~m}(\mathrm{C}=\mathrm{C}), 1594 \mathrm{~m}(\mathrm{C}=\mathrm{N})$,
Table 1. Crystallographic data for compound 2c

\begin{tabular}{l|l}
\hline \multicolumn{1}{c|}{ Parameter } & \multicolumn{1}{c}{ 2c } \\
\hline Formula & $\mathrm{C}_{13} \mathrm{H}_{8} \mathrm{Cl}_{2} \mathrm{~N}_{4} \mathrm{~S}$ \\
$T, \mathrm{~K}$ & 100 \\
$\lambda, \AA$ & 0.71073 \\
Crystal system & Monoclinic \\
Space group & $C 2 / c$ \\
$a, \AA$ & $19.1797(3)$ \\
$b, \AA$ & $7.75882(2)$ \\
$c, \AA$ & $18.4182(2)$ \\
$\beta$, deg & $97.3825(9)$ \\
$V, \AA^{3}$ & $2718.12(6)$ \\
$Z$ & 8 \\
$d_{\text {calc }}, \mathrm{g} / \mathrm{cm}^{3}$ & 1.580 \\
$\mu\left(\right.$ Mo $\left.K_{\alpha}\right)$, mm $^{-1}$ & 0.624 \\
Crystal size, mm & $0.42 \times 0.41 \times 0.16$ \\
Reflections collected: & \\
$\quad$ all & 30050 \\
$\quad$ independent & $4162\left(R_{\text {int }} 0.0175\right)$ \\
Refined parameters & 181 \\
GOOF & 1.052 \\
$I>2 \sigma(I)$ & $R_{1} 0.0275, R_{2} 0.0719$ \\
$\quad$ All data & $R^{1} 0.0291, R_{2} 0.0730$ \\
\hline
\end{tabular}

$1560 \mathrm{~s}(\mathrm{Ph}), 1074 \mathrm{~m}(\mathrm{C}-\mathrm{N}), 736 \mathrm{~m}(\mathrm{C}-\mathrm{Cl}), 697 \mathrm{~s}(\mathrm{C}-\mathrm{S})$. ${ }^{1} \mathrm{H}$ NMR spectrum, $\delta$, ppm: $6.69 \mathrm{~s}(1 \mathrm{H}, \mathrm{CH}), 7.56-7.60$ $\mathrm{m}(5 \mathrm{H}, \mathrm{Ph}) .{ }^{13} \mathrm{C}$ NMR spectrum, $\delta_{\mathrm{C}}, \mathrm{ppm}: 122.8(\mathrm{SC}=)$, $124.6(=\mathrm{CCl}), 126.8(\mathrm{Ph}), 129.9(\mathrm{Ph}), 130.9(\mathrm{Ph}), 133.3$ $(\mathrm{Ph}), 148.5(\mathrm{C}-\mathrm{N})$. Found, \%: C 39.45; H 2.33; N 20.44. $\mathrm{C}_{9} \mathrm{H}_{6} \mathrm{Cl}_{2} \mathrm{~N}_{4} \mathrm{~S}$. Calculated, \%: C 39.58; H 2.21; N 20.51.

Compounds $\mathbf{2 b}-\mathbf{2 g}$ were prepared similarly.

(E)-5-[(1,2-Dichlorovinyl)thio]-1-[2-(difluoromethoxy)phenyl]-1 $\boldsymbol{H}$-tetrazole (2b). Yield 49\%, yellow

Scheme 2.

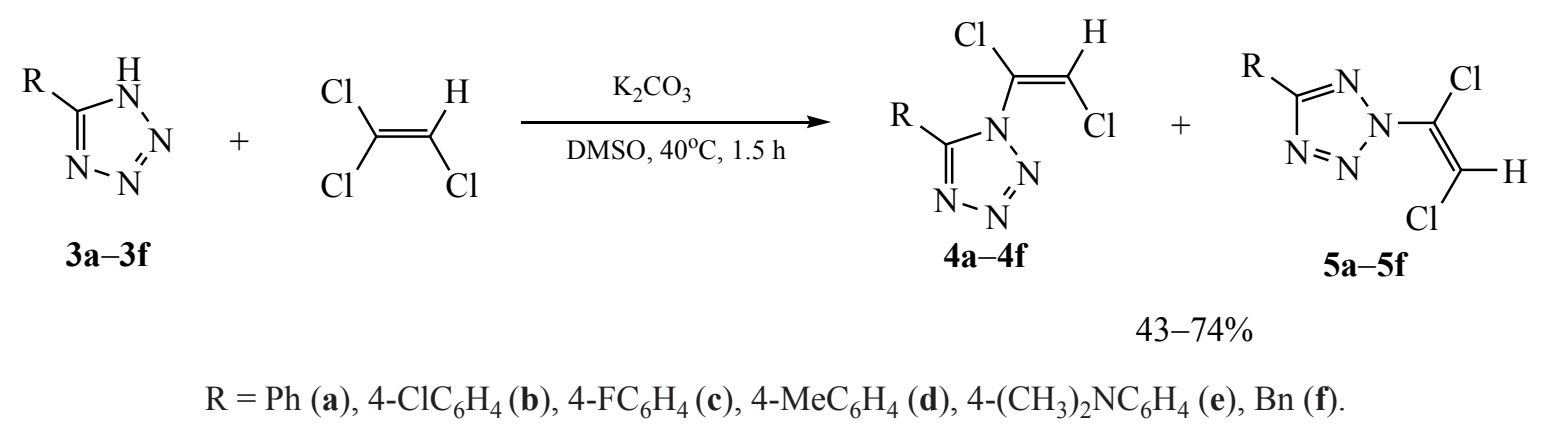


oil. IR spectrum, $v, \mathrm{~cm}^{-1}(\mathrm{KBr}): 3159 \mathrm{~m}(\mathrm{CH}), 3029 \mathrm{~m}$ $(\mathrm{C}=\mathrm{C}), 1598 \mathrm{~m}(\mathrm{C}=\mathrm{N}), 1510 \mathrm{~s}(\mathrm{Ph}), 1246 \mathrm{~m}\left(\mathrm{CF}_{2}\right), 1135 \mathrm{~s}$ (C-O), $1060 \mathrm{~m}(\mathrm{C}-\mathrm{N}), 760 \mathrm{~m}(\mathrm{C}-\mathrm{Cl}), 695 \mathrm{~s}(\mathrm{C}-\mathrm{S}) .{ }^{1} \mathrm{H}$ NMR spectrum, $\delta$, ppm: $6.48 \mathrm{~s}\left(1 \mathrm{H}, \mathrm{CHF}_{2}\right), 6.67 \mathrm{~s}(1 \mathrm{H}$, $\mathrm{CH}), 7.40-7.51 \mathrm{~m}(3 \mathrm{H}, \mathrm{Ph}), 7.62-7.66 \mathrm{~m}(1 \mathrm{H}, \mathrm{Ph}) .{ }^{13} \mathrm{C}$ NMR spectrum, $\delta_{\mathrm{C}}$, ppm: $115.20 \mathrm{t}\left(\mathrm{CHF}_{2}, J_{\mathrm{HH}} 264.5 \mathrm{~Hz}\right)$, $120.44(\mathrm{OCPh}), 122.98$ (=CCl), $124.57(\mathrm{SC}=), 126.33$ $(\mathrm{Ph}), 126.67(\mathrm{Ph}), 128.44(\mathrm{Ph}), 133.10(\mathrm{Ph}), 145.39(\mathrm{Ph})$, $150.26\left(\mathrm{C}_{\text {Tetr }}\right)$. Found, \%: C 35.60; H 1.59; N 16.41 . $\mathrm{C}_{10} \mathrm{H}_{6} \mathrm{Cl}_{2} \mathrm{~F}_{2} \mathrm{~N}_{4} \mathrm{OS}$. Calculated, \%: C 35.42; H 1.78; N 16.52 .

(E)-5-[(1,2-Dichlorovinyl)thio]-1-(naphth-1-yl)$1 \mathrm{H}$-tetrazole (2c). Yield 79\%, colorless crystals, mp $106-108^{\circ} \mathrm{C}\left(\mathrm{EtOH}-\mathrm{H}_{2} \mathrm{O}\right)$. IR spectrum, $v, \mathrm{~cm}^{-1}(\mathrm{KBr})$ : $3104 \mathrm{~m}(\mathrm{CH}), 3060 \mathrm{~m}(\mathrm{C}=\mathrm{C}), 1598 \mathrm{~m}(\mathrm{C}=\mathrm{N}), 1511 \mathrm{~s}$ (Ar), $1465 \mathrm{~s}(\mathrm{Ar}), 1058 \mathrm{~m}(\mathrm{C}-\mathrm{N}), 727 \mathrm{~m}(\mathrm{C}-\mathrm{Cl}), 698 \mathrm{~s}$ (C-S). ${ }^{1} \mathrm{H}$ NMR spectrum, $\delta$, ppm: $6.58 \mathrm{~s}(1 \mathrm{H}, \mathrm{CH}), 7.27 \mathrm{~d}$ $\left(1 \mathrm{H}, \mathrm{H}_{\mathrm{Ar}}, J_{\mathrm{HH}} 4.0 \mathrm{~Hz}\right), 7.55-7.64 \mathrm{~m}\left(4 \mathrm{H}, \mathrm{H}_{\mathrm{Ar}}\right), 7.99 \mathrm{~d}$ $\left(1 \mathrm{H}, \mathrm{H}_{\mathrm{Ar}}, J_{\mathrm{HH}} 4.0 \mathrm{~Hz}\right), 8.11 \mathrm{~d}\left(1 \mathrm{H}, \mathrm{H}_{\mathrm{Ar}}, J_{\mathrm{HH}} 4.0 \mathrm{~Hz}\right)$. ${ }^{13} \mathrm{C}$ NMR spectrum, $\delta_{\mathrm{C}}$, ppm: $121.77(\mathrm{Ar}), 122.6(\mathrm{Ar})$, 125.01 $(=\mathrm{CCl}), 125.05(\mathrm{SC}=), 125.2(\mathrm{Ar}), 127.3(\mathrm{Ar})$, 127.7 (Ar), 128.6 (Ar), 128.7 (Ar), 129.0 (Ar), 132.17 (Ar), 134.3 (Ar), 150.8 (C-N). Found, \%: C 48.46; H 2.59; N 17.22. $\mathrm{C}_{13} \mathrm{H}_{8} \mathrm{Cl}_{2} \mathrm{~N}_{4} \mathrm{~S}$. Calculated, \%: C 48.31; $\mathrm{H}$ $2.50 ; \mathrm{N} 17.34$.

(E)-5-[(1,2-Dichlorovinyl)thio]-1-benzyl-1 $H$ tetrazole (2d). Yield (80\%), yellow crystals, $\mathrm{mp} 56-58^{\circ} \mathrm{C}$ (hexane- $\left.\mathrm{CCl}_{4}\right)$. IR spectrum, $\mathrm{v}, \mathrm{cm}^{-1}(\mathrm{KBr}): 3105 \mathrm{~m}$ $(\mathrm{CH}), 3039 \mathrm{~m}(\mathrm{C}=\mathrm{C}), 2923 \mathrm{~m}\left(\mathrm{CH}_{2}\right), 1595 \mathrm{~m}(\mathrm{C}=\mathrm{N})$, $1453 \mathrm{~s}(\mathrm{Ph}), 1078 \mathrm{~m}(\mathrm{C}-\mathrm{N}), 748 \mathrm{~m}(\mathrm{C}-\mathrm{Cl}), 691 \mathrm{~s}(\mathrm{C}-\mathrm{S})$. ${ }^{1} \mathrm{H}$ NMR spectrum, $\delta$, ppm: $5.57 \mathrm{~s}\left(2 \mathrm{H}, \mathrm{CH}_{2}\right), 6.66 \mathrm{~s}$ $(1 \mathrm{H}, \mathrm{CH}), 7.27-7.29 \mathrm{~m}(2 \mathrm{H}, \mathrm{Ph}), 7.33-7.37 \mathrm{~m}(3 \mathrm{H}, \mathrm{Ph})$. ${ }^{13} \mathrm{C} \mathrm{NMR}$ spectrum, $\delta_{\mathrm{C}}$, ppm: $52.0\left(\mathrm{CH}_{2}\right), 123.4(=\mathrm{CCl})$, $124.8(\mathrm{SC}=), 128.2(\mathrm{Ph}), 129.35(\mathrm{Ph}), 129.39(\mathrm{Ph}), 132.59$ (Ph), 147.70 (C-N). Found, \%: 41.67; H 2.95; N 19.40. $\mathrm{C}_{10} \mathrm{H}_{8} \mathrm{Cl}_{2} \mathrm{~N}_{4} \mathrm{~S}$. Calculated, \%: C 41.83; H 2.81; N 19.51 .

(E)-5-[(1,2-Dichlorovinyl)thio]-1-phenetyl-1 Htetrazole (2e). Yield 55\%, yellow oil. IR spectrum, $v, \mathrm{~cm}^{-1}(\mathrm{KBr}): 3109 \mathrm{~m}(\mathrm{CH}), 3039 \mathrm{~m}(\mathrm{C}=\mathrm{C}), 2923 \mathrm{~m}$ $\left(\mathrm{CH}_{2}\right), 1559 \mathrm{~m}(\mathrm{C}=\mathrm{N}), 1492 \mathrm{~s}(\mathrm{Ph}), 1078 \mathrm{~m}(\mathrm{C}-\mathrm{N}), 748 \mathrm{~m}$ $(\mathrm{C}-\mathrm{Cl}), 670 \mathrm{~s}(\mathrm{C}-\mathrm{S}) .{ }^{1} \mathrm{H}$ NMR spectrum, $\delta$, ppm: $3.23 \mathrm{t}$ $\left(2 \mathrm{H}, \mathrm{CH}_{2}, J_{\mathrm{HH}} 8.0 \mathrm{~Hz}\right), 4.60 \mathrm{t}\left(2 \mathrm{H}, \mathrm{CH}_{2}, J_{\mathrm{HH}} 8.0 \mathrm{~Hz}\right), 6.66 \mathrm{~s}$ $(1 \mathrm{H}, \mathrm{CH}), 7.04 \mathrm{~d}\left(2 \mathrm{H}, \mathrm{Ph}, J_{\mathrm{HH}} 8.0 \mathrm{~Hz}\right), 7.24-7.29 \mathrm{~m}$ $(3 \mathrm{H}, \mathrm{Ph}) .{ }^{13} \mathrm{C}$ NMR spectrum, $\delta_{\mathrm{C}}, \mathrm{ppm}: 35.97\left(\mathrm{CH}_{2}\right)$, $49.61\left(\mathrm{CH}_{2}\right), 124.65(=\mathrm{CCl}), 127.65(\mathrm{SC}=), 128.85(\mathrm{Ph})$, $129.16(\mathrm{Ph}), 135.79(\mathrm{Ph}), 147.88(\mathrm{C}-\mathrm{N})$. Found, \%: C 44.02; H 3.24; $\mathrm{N}$ 18.71. $\mathrm{C}_{11} \mathrm{H}_{10} \mathrm{Cl}_{2} \mathrm{~N}_{4} \mathrm{~S}$. Calculated, \%: C 43.87; H 3.35; N 18.60.
(E)-5-[(1,2-Dichlorovinyl)thio]-1-cyclohexyl-1Htetrazole (2f). The product was purified by column chromatography in the ethyl acetate-hexane system. (2: 8). Yield $42 \%$, yellow crystals, $\mathrm{mp} 68-70^{\circ} \mathrm{C}$ (hexane). IR spectrum, $v, \mathrm{~cm}^{-1}(\mathrm{KBr}): 3107 \mathrm{~m}(\mathrm{CH}), 3021 \mathrm{~m}(\mathrm{C}=\mathrm{C})$, $2944 \mathrm{~m}\left(\mathrm{CH}_{2}\right), 1560 \mathrm{~m}(\mathrm{C}=\mathrm{N}), 1348 \mathrm{~s}\left[\left(\mathrm{CH}_{2}\right)_{n}\right], 1075 \mathrm{~m}$ $(\mathrm{C}-\mathrm{N}), 755 \mathrm{~m}(\mathrm{C}-\mathrm{Cl}), 610 \mathrm{~s}(\mathrm{C}-\mathrm{S}) .{ }^{1} \mathrm{H}$ NMR spectrum, $\delta$, ppm: $1.23-1.48 \mathrm{~m}\left(4 \mathrm{H}, \mathrm{CH}_{2}\right), 1.43 \mathrm{~d}\left(2 \mathrm{H}, \mathrm{CH}_{2}, J_{\mathrm{HH}}\right.$ $6.0 \mathrm{~Hz}), 1.94-2.02 \mathrm{~m}\left(4 \mathrm{H}, \mathrm{CH}_{2}\right), 4.37-4.43 \mathrm{~m}(1 \mathrm{H}, \mathrm{CH})$, $6.75 \mathrm{~s}(1 \mathrm{H}, \mathrm{CH}) .{ }^{13} \mathrm{C}$ NMR spectrum, $\delta_{\mathrm{C}}, \mathrm{ppm}: 24.8$ $\left(\mathrm{CH}_{2}\right), 25.3\left(\mathrm{CH}_{2}\right), 32.7\left(\mathrm{CH}_{2}\right), 59.2(\mathrm{CH}), 124.0(=\mathrm{CCl})$, $124.6(\mathrm{SC}=), 146.3(\mathrm{C}-\mathrm{N})$. Found, \%: C 38.91; H 4.15; $\mathrm{N} 20.28 ; \mathrm{S} 11.62 . \mathrm{C}_{9} \mathrm{H}_{12} \mathrm{Cl}_{2} \mathrm{~N}_{4} \mathrm{~S}$ Calculated, \%: C 38.72; $\mathrm{H} 4.33$; N 20.07; S 11.48.

(E)-5-[(1,2-Dichlorovinyl)thio]-1-methyl-1 Htetrazole (2g). Yield $43 \%$, yellow crystals, $\mathrm{mp} 48-50^{\circ} \mathrm{C}$ (EtOAc). IR spectrum, $v, \mathrm{~cm}^{-1}(\mathrm{KBr}): 3121 \mathrm{~m}(\mathrm{CH})$, $3060 \mathrm{~m}(\mathrm{C}=\mathrm{C}), 2956 \mathrm{~m}\left(\mathrm{CH}_{3}\right), 1565 \mathrm{~m}(\mathrm{C}=\mathrm{N}), 1089 \mathrm{~m}$ $(\mathrm{C}-\mathrm{N}), 735 \mathrm{~m}(\mathrm{C}-\mathrm{Cl}), 670 \mathrm{~s}(\mathrm{C}-\mathrm{S}) .{ }^{1} \mathrm{H}$ NMR spectrum, $\delta$, ppm: $4.08 \mathrm{~s}\left(3 \mathrm{H}, \mathrm{CH}_{3}\right), 6.74 \mathrm{~s}(1 \mathrm{H}, \mathrm{CH}) .{ }^{13} \mathrm{C} \mathrm{NMR}$ spectrum, $\delta_{\mathrm{C}}$, ppm: $34.61\left(\mathrm{CH}_{3}\right), 123.36(=\mathrm{CCl}), 124.51$ $(\mathrm{SC}=), 147.75(\mathrm{C}-\mathrm{N})$. Found, \%: C 22.59; H 2.13; N 26.68. $\mathrm{C}_{4} \mathrm{H}_{4} \mathrm{Cl}_{2} \mathrm{~N}_{4} \mathrm{~S}$. Calculated, \%: C 22.76; H 1.91; N 26.55 .

(E)-1-(1,2-Dichlorovinyl)-5-phenyl-1H-tetrazole (4a) and (E)-2-(1,2-dichlorovinyl)-5-phenyl-2 $H$ tetrazole (5a). Trichlorethylene $1.35 \mathrm{~g}(10.27 \mathrm{mmol})$ was added to a solution of $1 \mathrm{~g}(6.85 \mathrm{mmol}) 5$-phenyl$1 \mathrm{H}$-tetrazole and $1.15 \mathrm{~g}(20.55 \mathrm{mmol})$ of $\mathrm{KOH}$ in $20 \mathrm{~mL}$ of DMSO. The reaction mixture was stirred for $1.5 \mathrm{~h}$ at $40^{\circ} \mathrm{C}$, then poured into $100 \mathrm{~mL}$ of chloroform and extracted with cold water $(3 \times 30 \mathrm{~mL})$. The organic layer was washed with saturated $\mathrm{NaCl}$ solution, then dried with $\mathrm{Na}_{2} \mathrm{SO}_{4}$. The solvent was removed in vacuum. The product was purified by column chromatography in the ethyl acetate-hexane system (1:9). Yield $1.22 \mathrm{~g} \mathrm{(74 \% )}$ $(\mathbf{4 a}: \mathbf{5 a}=1: 1.2$, by NMR).

(E)-1-(1,2-Dichlorovinyl)-5-phenyl-1H-tetrazole (4a). Yellowish crystals, mp $63-65^{\circ} \mathrm{C}$ (hexane). IR spectrum, $v, \mathrm{~cm}^{-1}(\mathrm{KBr}): 3093 \mathrm{~m}(\mathrm{CH}), 3013 \mathrm{~m}(\mathrm{C}=\mathrm{C})$, $1607 \mathrm{~m}(\mathrm{C}=\mathrm{N}), 1531 \mathrm{~s}(\mathrm{Ph}), 1079 \mathrm{~m}(\mathrm{C}-\mathrm{N}), 733 \mathrm{~m}(\mathrm{C}-\mathrm{Cl})$. ${ }^{1} \mathrm{H}$ NMR spectrum, $\delta$, ppm $\left(\mathrm{CDCl}_{3}\right): 6.82 \mathrm{~s}(1 \mathrm{H}, \mathrm{CH})$, 7.63-7.53 m (3H, Ph), 7.87-7.85 m $(2 \mathrm{H}, \mathrm{Ph}) .{ }^{13} \mathrm{C}$ NMR spectrum, $\delta_{\mathrm{C}}$, ppm: $122.3(=\mathrm{CCl}), 122.5(\mathrm{~N}-\mathrm{C}=)$, $123.0(\mathrm{Ph}), 128.3(\mathrm{Ph}), 129.5(\mathrm{Ph}), 132.4(\mathrm{Ph}), 154.3$ (C-N). Found, \%: C 44.75; H 2.58; N 23.18; $\mathrm{C}_{9} \mathrm{H}_{6} \mathrm{Cl}_{2} \mathrm{~N}_{4}$. Calculated, \%: C 44.84; H 2.51; N 23.24. 
(E)-2-(1,2-Dichlorovinyl)-5-phenyl-2H-tetrazole (5a). Yellow oil. IR spectrum, $v, \mathrm{~cm}^{-1}(\mathrm{KBr}): 3080 \mathrm{~m}$ $(\mathrm{CH}), 3034 \mathrm{~m}(\mathrm{C}=\mathrm{C}), 1620 \mathrm{~m}(\mathrm{C}=\mathrm{N}), 1530 \mathrm{~s}(\mathrm{Ph}), 1078 \mathrm{~m}$ $(\mathrm{C}-\mathrm{N}), 731 \mathrm{~m}(\mathrm{C}-\mathrm{Cl}) .{ }^{1} \mathrm{H}$ NMR spectrum, $\delta$, ppm: $6.82 \mathrm{~s}$ $(1 \mathrm{H}, \mathrm{CH}), 7.50-7.54 \mathrm{~m}(3 \mathrm{H}, \mathrm{Ph}), 8.21-8.23 \mathrm{~m}(2 \mathrm{H}, \mathrm{Ph})$. ${ }^{13} \mathrm{C}$ NMR spectrum, $\delta_{\mathrm{C}}$, ppm: $119.1(=\mathrm{CCl}), 124.7(\mathrm{Ph})$, $126.3(\mathrm{~N}-\mathrm{C}=), 127.4(\mathrm{Ph}), 129.2(\mathrm{Ph}), 131.2(\mathrm{Ph}), 165.5$ $(\mathrm{C}-\mathrm{N})$. Found, \%: C 44.96; H 2.45; N 23.32; $\mathrm{C}_{9} \mathrm{H}_{6} \mathrm{Cl}_{2} \mathrm{~N}_{4}$. Calculated, \%: C 44.84; H 2.51; N 23.24.

Compounds $\mathbf{4 b}-\mathbf{4 f}$ and $\mathbf{5 b}-\mathbf{5 f}$ were prepared similarly.

(E)-5-(4-Chlororphenyl)-1-(1,2-dichlorovinyl)$1 H$-tetrazole (4b) and $(E)-5-(4-c h l o r o p h e n y l)-2-(1,2-$ dichlorovinyl)-2 $\boldsymbol{H}$-tetrazole (5b). Yield 43\% (4b : 5b $=$ $1: 1.7)$.

(E)-5-(4-Chlorophenyl)-1-(1,2-dichlorovinyl)-1 Htetrazole (4b). White crystals, $\mathrm{mp} 141-143^{\circ} \mathrm{C}$ (hexane). IR spectrum, $v, \mathrm{~cm}^{-1}(\mathrm{KBr}): 3082 \mathrm{~m}(\mathrm{CH}), 3012 \mathrm{~m}(\mathrm{C}=\mathrm{C})$, $1600 \mathrm{~m}(\mathrm{C}=\mathrm{N}), 1520 \mathrm{~s}(\mathrm{Ph}), 1089 \mathrm{~m}(\mathrm{C}-\mathrm{N}), 740 \mathrm{~m}(\mathrm{C}-\mathrm{Cl})$. ${ }^{1} \mathrm{H}$ NMR spectrum, $\delta$, ppm: $6.88 \mathrm{~s}(1 \mathrm{H}, \mathrm{CH}), 7.55-7.59 \mathrm{~m}$ $(2 \mathrm{H}, \mathrm{Ph}), 7.83-7.86 \mathrm{~m}(2 \mathrm{H}, \mathrm{Ph}) .{ }^{13} \mathrm{C}$ NMR spectrum, $\delta_{\mathrm{C}}$, ppm: $120.9(=\mathrm{CCl}), 122.5(\mathrm{~N}-\mathrm{C}=), 122.8(\mathrm{Ph}), 129.5$ $(\mathrm{Ph}), 129.9(\mathrm{Ph}), 138.9(\mathrm{Ph}), 153.3(\mathrm{C}-\mathrm{N})$. Found, \%: C $39.15 ; \mathrm{H} 1.73 ; \mathrm{N} 20.46 ; \mathrm{C}_{9} \mathrm{H}_{5} \mathrm{Cl}_{3} \mathrm{~N}_{4}$. Calculated, \%: C 39.23 ; H 1.83; N 20.34.

(E)-5-(4-Chlorophenyl)-2-(1,2-dichlorovinyl)-2 Htetrazole (5b). White crystals, $\mathrm{mp} 81-83^{\circ} \mathrm{C}$ (hexane). IR spectrum, $v, \mathrm{~cm}^{-1}(\mathrm{KBr}): 3096 \mathrm{~m}(\mathrm{CH}), 3025 \mathrm{~m}(\mathrm{C}=\mathrm{C})$, $1628 \mathrm{~m}(\mathrm{C}=\mathrm{N}), 1545 \mathrm{~s}(\mathrm{Ph}), 1068 \mathrm{~m}(\mathrm{C}-\mathrm{N}), 742 \mathrm{~m}(\mathrm{C}-\mathrm{Cl})$. ${ }^{1} \mathrm{H}$ NMR spectrum, $\delta$, ppm: $6.85 \mathrm{~s}(1 \mathrm{H}, \mathrm{CH}), 7.50-7.54 \mathrm{~m}$ $(3 \mathrm{H}, \mathrm{Ph}), 8.16-8.19 \mathrm{~m}(2 \mathrm{H}, \mathrm{Ph}) .{ }^{13} \mathrm{C}$ NMR spectrum, $\delta_{\mathrm{C}}, \mathrm{ppm}: 119.1(=\mathrm{CCl}), 124.5(\mathrm{Ph}), 124.7(\mathrm{~N}-\mathrm{C}=), 128.6$ $(\mathrm{Ph}), 129.4(\mathrm{Ph}), 137.3(\mathrm{Ph}), 164.6(\mathrm{C}-\mathrm{N})$. Found, \%: C $39.06 ; \mathrm{H} 2.11 ; \mathrm{N} 20.32 ; \mathrm{C}_{9} \mathrm{H}_{5} \mathrm{Cl}_{3} \mathrm{~N}_{4}$. Calculated, \%: C 39.23; H 1.83; N 20.34.

(E)-5-(4-Fluorophenyl)-1-(1,2-dichlorovinyl)$1 H$-tetrazole $(4 c)$ and $(E)-5-(4-f l u o r o p h e n y l) 2-(1,2-$ dichlorovinyl)-2H-tetrazole (5c). Yield $87 \%(4 \mathrm{c}: 5 \mathrm{c}=$ $1: 2$ ).

(E)-5-(4-Fluorophenyl)-1-(1,2-dichlorovinyl)-1Htetrazole (4c). Colorless crystals, $\mathrm{mp} 84-86^{\circ} \mathrm{C}$ (hexane). IR spectrum, $v, \mathrm{~cm}^{-1}: 3097 \mathrm{~m}(\mathrm{CH}), 2992 \mathrm{~m}(\mathrm{C}=\mathrm{C}), 1608 \mathrm{~s}$ $(\mathrm{C}=\mathrm{N}), 1540 \mathrm{~m}(\mathrm{Ph}), 1091 \mathrm{~s}(\mathrm{C}-\mathrm{N}), 1022 \mathrm{~m}(\mathrm{C}-\mathrm{F}), 746 \mathrm{~m}$ (C-Cl). ${ }^{1} \mathrm{H}$ NMR spectrum, $\delta$, ppm: $6.87 \mathrm{~s}(1 \mathrm{H}, \mathrm{CH})$, 7.27-7.31 m (2H, Ph), 7.90-7.94 m (2H, Ph). ${ }^{13} \mathrm{C}$ NMR spectrum, $\delta_{\mathrm{C}}$, ppm: $116.8(=\mathrm{C}-\mathrm{Cl}), 117.0(\mathrm{~N}-\mathrm{C}=), 118.7$ $(\mathrm{Ph}), 122.4(\mathrm{Ph}), 122.9(\mathrm{Ph}), 130.6(\mathrm{Ph}), 153.3(\mathrm{C}-\mathrm{N})$.
Found, \%: C 41.79; $\mathrm{H} 1.80 ; \mathrm{N} 21.79 . \mathrm{C}_{9} \mathrm{H}_{5} \mathrm{Cl}_{2} \mathrm{FN}_{4}$. Calculated, \%: C 41.73; H 1.95; Cl 27.37; F 7.33; N 21.63.

(E)-5-(4-Fluorophenyl)-2-(1,2-dichlorovinyl)-2Htetrazole (5c). Yellowish crystals, $\mathrm{mp} 52-54^{\circ} \mathrm{C}$ (hexane). IR spectrum, $v, \mathrm{~cm}^{-1}: 3090 \mathrm{~m}(\mathrm{CH}), 2997 \mathrm{~m}(\mathrm{C}=\mathrm{C}), 1604 \mathrm{~s}$ $(\mathrm{C}=\mathrm{N}), 1541 \mathrm{~m}(\mathrm{Ph}), 1093 \mathrm{~s}(\mathrm{C}-\mathrm{N}), 1023 \mathrm{~m}(\mathrm{C}-\mathrm{F}), 757 \mathrm{~m}$ $(\mathrm{C}-\mathrm{Cl}) .{ }^{1} \mathrm{H}$ NMR spectrum, $\delta$, ppm: $6.85 \mathrm{~s}(1 \mathrm{H}, \mathrm{CH})$, 7.22-7.27 m (2H, Ph), 8.23-8.27 m (2H, Ph). ${ }^{13} \mathrm{C}$ NMR spectrum, $\delta_{\mathrm{C}}$, ppm: $116.4(=\mathrm{CCl}), 119.0(\mathrm{~N}-\mathrm{C}=), 122.5$ $(\mathrm{Ph}), 124.6(\mathrm{Ph}), 129.4(\mathrm{Ph}), 129.5(\mathrm{Ph}), 163.3(\mathrm{C}-\mathrm{N})$. Found, \%: C 41.61; $\mathrm{H} 1.89 ; \mathrm{N} 21.45 . \mathrm{C}_{9} \mathrm{H}_{5} \mathrm{Cl}_{2} \mathrm{FN}_{4}$. Calculated, \%: C 41.73; H 1.95; Cl 27.37; F 7.33; N 21.63.

(E)-5-(p-Tolyl)-1-(1,2-dichlorovinyl)-1 H-tetrazole (4d) and (E)-5-(p-tolyl)-2-(1,2-dichlorovinyl)-2 $H$ tetrazole (5d). Yield $65 \%(\mathbf{4 d}: \mathbf{5 d}=1.3: 1)$.

(E)-5-(p-Tolyl)-1-(1,2-dichlorovinyl)-1 $H$-tetrazole (4d). Colorless crystals, mp $84-86^{\circ} \mathrm{C}\left(\mathrm{EtOH}-\mathrm{H}_{2} \mathrm{O}\right)$. IR spectrum, $v, \mathrm{~cm}^{-1}(\mathrm{KBr}): 3077 \mathrm{~m}(\mathrm{CH}), 3036 \mathrm{~m}(\mathrm{C}=\mathrm{C})$, $2859 \mathrm{~s}\left(\mathrm{CH}_{3}\right), 1612 \mathrm{~s}(\mathrm{C}=\mathrm{N}), 1535 \mathrm{~s}(\mathrm{Ph}), 1090 \mathrm{~m}(\mathrm{C}-\mathrm{N})$, $736 \mathrm{~m}(\mathrm{C}-\mathrm{Cl}) .{ }^{1} \mathrm{H}$ NMR spectrum, $\delta$, ppm: $2.42 \mathrm{~s}(3 \mathrm{H}$, $\left.\mathrm{CH}_{3}\right), 7.48-7.50 \mathrm{~m}(2 \mathrm{H}, \mathrm{Ph}), 7.76-7.78 \mathrm{~m}(2 \mathrm{H}, \mathrm{Ph}), 7.82 \mathrm{~s}$ $(1 \mathrm{H}, \mathrm{CH}) .{ }^{13} \mathrm{C}$ NMR spectrum, $\delta_{\mathrm{C}}, \mathrm{ppm}: 21.6\left(\mathrm{CH}_{3}\right)$, $119.2(=\mathrm{CCl}), 121.6(\mathrm{~N}-\mathrm{C}=), 124.8(\mathrm{Ph}), 128.3(\mathrm{Ph})$, $130.8(\mathrm{Ph}), 143.6(\mathrm{Ph}), 154.2(\mathrm{C}-\mathrm{N})$. Found, \%: C 47.15; $\mathrm{H}$ 3.04; $\mathrm{N}$ 21.85. $\mathrm{C}_{10} \mathrm{H}_{8} \mathrm{Cl}_{2} \mathrm{~N}_{4}$. Calculated, \%: C 47.08; H 3.16; N 21.96.

(E)-5-(p-Tolyl)-2-(1,2-dichlorovinyl)-2H-tetrazole (5d). Yellowish crystals, mp $88-91{ }^{\circ} \mathrm{C}\left(\mathrm{EtOH}-\mathrm{H}_{2} \mathrm{O}\right)$. IR spectrum, $v, \mathrm{~cm}^{-1}(\mathrm{KBr}): 3088 \mathrm{~m}(\mathrm{CH}), 3056 \mathrm{~m}(\mathrm{C}=\mathrm{C})$, $2855 \mathrm{~s}\left(\mathrm{CH}_{3}\right), 1611 \mathrm{~s}(\mathrm{C}=\mathrm{N}), 1565 \mathrm{~m}(\mathrm{Ph}), 1071 \mathrm{~m}(\mathrm{C}-\mathrm{N})$, $742 \mathrm{~m}(\mathrm{C}-\mathrm{Cl}) .{ }^{1} \mathrm{H}$ NMR spectrum, $\delta$, ppm: $2.40 \mathrm{~s}(3 \mathrm{H}$, $\left.\mathrm{CH}_{3}\right), 7.41-7.44 \mathrm{~m}(2 \mathrm{H}, \mathrm{Ph}), 7.85 \mathrm{~s}(1 \mathrm{H}, \mathrm{CH}), 8.02-8.04 \mathrm{~m}$ $(2 \mathrm{H}, \mathrm{Ph}) .{ }^{13} \mathrm{C}$ NMR spectrum, $\delta_{\mathrm{C}}, \mathrm{ppm}: 21.6\left(\mathrm{CH}_{3}\right), 122.0$ $(=\mathrm{CCl}), 123.1(\mathrm{~N}-\mathrm{C}=), 123.3(\mathrm{Ph}), 127.3(\mathrm{Ph}), 130.5$ $(\mathrm{Ph}), 142.1(\mathrm{Ph}), 165.4(\mathrm{C}-\mathrm{N})$. Found, \%: C 46.94; H 3.25; $\mathrm{N}$ 22.02. $\mathrm{C}_{10} \mathrm{H}_{8} \mathrm{Cl}_{2} \mathrm{~N}_{4}$. Calculated, \%: $\mathrm{C} 47.08 ; \mathrm{H}$ 3.16 ; N 21.96.

(E)-4-[1-(1,2-Dichlorovinyl)-1H-tetrazol-5-yl)- $\mathrm{N}, \mathrm{N}$ dimethylaniline (4e) and $(E)$-4-[2-(1,2-dichlorovinyl)2H-tetrazol-5-yl]- $\mathrm{N}, \mathrm{N}$-dimethylaniline (5e). Yield $67 \%$ $(\mathbf{4 d}: \mathbf{5 d}=1: 1.7)$.

(E)-4-[1-(1,2-Dichlorovinyl)-1H-tetrazole-5-yl]$\boldsymbol{N}, \boldsymbol{N}$-dimethylaniline (4e). Yellowish crystals, $\mathrm{mp}$ $76-78^{\circ} \mathrm{C}(\mathrm{EtOH})$. IR spectrum, $v, \mathrm{~cm}^{-1}(\mathrm{KBr}): 3092 \mathrm{~m}$ $(\mathrm{CH}), 3035 \mathrm{~m}(\mathrm{C}=\mathrm{C}), 2823 \mathrm{~s}\left(\mathrm{CH}_{3}\right), 1611 \mathrm{~s}(\mathrm{C}=\mathrm{N}), 1544 \mathrm{~s}$ $(\mathrm{Ph}), 1295\left(\mathrm{R}_{2} \mathrm{~N}\right), 1074 \mathrm{~m}(\mathrm{C}-\mathrm{N}), 744 \mathrm{~m}(\mathrm{C}-\mathrm{Cl}) .{ }^{1} \mathrm{H} \mathrm{NMR}$ spectrum, $\delta$, ppm: $3.10 \mathrm{~s}\left(6 \mathrm{H}, \mathrm{CH}_{3}\right), 6.79-6.81 \mathrm{~m}(2 \mathrm{H}$, 
$\mathrm{Ph}), 6.84 \mathrm{~s}(1 \mathrm{H}, \mathrm{CH}), 7.81-7.84 \mathrm{~m}(2 \mathrm{H}, \mathrm{Ph}) .{ }^{13} \mathrm{C} \mathrm{NMR}$ spectrum, $\delta_{\mathrm{C}}$, ppm: $40.1\left(\mathrm{CH}_{3}\right), 108.8(\mathrm{Ph}), 111.9(=\mathrm{CCl})$, $121.8(\mathrm{~N}-\mathrm{C}=), 123.8(\mathrm{Ph}), 129.4(\mathrm{Ph}), 152.5(\mathrm{Ph}), 154.2$ (C-N). Found, \%: C 46.55; H 3.85; N 24.73. $\mathrm{C}_{11} \mathrm{H}_{11} \mathrm{Cl}_{2} \mathrm{~N}_{5}$. Calculated, \%: C 46.50; H 3.90; N 24.65.

(E)-4-[2-(1,2-Dichlorovinyl)-2H-tetrazol-5-yl)$\mathrm{N}, \mathrm{N}$-dimethylaniline (5e). Yellow crystals, $\mathrm{mp} 60-62^{\circ} \mathrm{C}$ (EtOH). IR spectrum, $v, \mathrm{~cm}^{-1}(\mathrm{KBr}): 3088 \mathrm{~m}(\mathrm{CH}), 3062 \mathrm{~m}$ $(\mathrm{C}=\mathrm{C}), 2820 \mathrm{~s}\left(\mathrm{CH}_{3}\right), 1607 \mathrm{~s}(\mathrm{C}=\mathrm{N}), 1521 \mathrm{~s}(\mathrm{Ph}), 1275$ $\left(\mathrm{R}_{2} \mathrm{~N}\right), 1063 \mathrm{~m}(\mathrm{C}-\mathrm{N}), 750 \mathrm{~m}(\mathrm{C}-\mathrm{Cl}) .{ }^{1} \mathrm{H}$ NMR spectrum, $\delta$, ppm: $3.09 \mathrm{~s}\left(6 \mathrm{H}, \mathrm{CH}_{3}\right), 6.80 \mathrm{~s}(1 \mathrm{H}, \mathrm{CH}), 6.87-6.89 \mathrm{~m}$ $(2 \mathrm{H}, \mathrm{Ph}), 8.10-8.12 \mathrm{~m}(2 \mathrm{H}, \mathrm{Ph}) .{ }^{13} \mathrm{C}$ NMR spectrum, $\delta_{\mathrm{C}}$, ppm: $40.1\left(\mathrm{CH}_{3}\right), 100.1(\mathrm{Ph}), 113.1(=\mathrm{CCl}), 118.4$ $(\mathrm{N}-\mathrm{C}=), 128.5(\mathrm{Ph}), 133.6(\mathrm{Ph}), 142.9(\mathrm{Ph}), 165.8(\mathrm{C}-\mathrm{N})$. Found, \%: C 45.68; $\mathrm{H}$ 3.99; $\mathrm{N}$ 21.51. $\mathrm{C}_{11} \mathrm{H}_{11} \mathrm{Cl}_{2} \mathrm{~N}_{5}$. Calculated, \%: C 46.50; H 3.90; N 24.65.

(E)-1-(1,2-Dichlorovinyl)-5-benzyl-1H-tetrazole (4f) and (E)-2-(1,2-dichlorovinyl)-5-benzyl-1 $H$ tetrazole (5f). Yield 47\% (4f: $\mathbf{5 f}=1: 1.2)$.

(E)-1-(1,2-Dichlorovinyl)-5-benzyl-1H-tetrazole (4f). Colorless crystals, $\mathrm{mp} 56-58^{\circ} \mathrm{C}$ (hexane-EtOAc). IR spectrum, $v, \mathrm{~cm}^{-1}$ : $3093 \mathrm{~s}(\mathrm{CH}), 3031 \mathrm{~m}(\mathrm{C}=\mathrm{C}), 2913 \mathrm{~m}$ $\left(\mathrm{CH}_{2}\right), 1622 \mathrm{~m}(\mathrm{C}=\mathrm{N}), 1502 \mathrm{~s}(\mathrm{Ph}), 1094 \mathrm{~m}(\mathrm{C}-\mathrm{N}), 720 \mathrm{~m}$ $(\mathrm{C}-\mathrm{Cl}) .{ }^{1} \mathrm{H}\left(\mathrm{CDCl}_{3}\right)$ NMR spectrum, $\delta$, ppm: $4.34 \mathrm{~s}(2 \mathrm{H}$, $\left.\mathrm{CH}_{2}\right), 6.71 \mathrm{~s}(1 \mathrm{H}, \mathrm{CH}), 7.25-7.27 \mathrm{~m}(3 \mathrm{H}, \mathrm{Ph}), 7.33-7.35 \mathrm{~m}$ $(2 \mathrm{H}, \mathrm{Ph}) .{ }^{13} \mathrm{C}$ NMR spectrum, $\delta_{\mathrm{C}}, \mathrm{ppm}: 29.7\left(\mathrm{CH}_{2}\right), 121.9$ $(=\mathrm{CCl}), 127.9(\mathrm{~N}-\mathrm{C}=), 128.9(\mathrm{Ph}), 129.0(\mathrm{Ph}), 132.6$ $(\mathrm{Ph}), 142.8(\mathrm{Ph}), 154.6(\mathrm{C}-\mathrm{N})$. Found, \%: C 47.23; H 3.30; N 21.79. $\mathrm{C}_{10} \mathrm{H}_{8} \mathrm{Cl}_{2} \mathrm{~N}_{4}$. Calculated, \%: C 47.08; $\mathrm{H}$ 3.16 ; N 21.96.

(E)-2-(1,2-Dichlorovinyl)-5-benzyl-1 $H$-tetrazole (4f). Yellow oil. IR spectrum, $v, \mathrm{~cm}^{-1}: 3086 \mathrm{~s}(\mathrm{CH}), 3028$ $\mathrm{m}(\mathrm{C}=\mathrm{C}), 2957 \mathrm{~m}\left(\mathrm{CH}_{2}\right), 1627 \mathrm{~s}(\mathrm{C}=\mathrm{N}), 1496 \mathrm{~m}(\mathrm{Ph})$, $1086 \mathrm{~m}(\mathrm{C}-\mathrm{N}), 737 \mathrm{~m}(\mathrm{C}-\mathrm{Cl}) .{ }^{1} \mathrm{H}$ NMR spectrum, $\delta$, ppm: $4.36 \mathrm{~s}\left(2 \mathrm{H}, \mathrm{CH}_{2}\right), 6.78 \mathrm{~s}(1 \mathrm{H}, \mathrm{CH}), 7.30-7.32 \mathrm{~m}(3 \mathrm{H}, \mathrm{Ph})$, 7.35-7.37 m (2H, Ph). ${ }^{13} \mathrm{C}$ NMR spectrum, $\delta_{\mathrm{C}}$, ppm: 31.8 $\left(\mathrm{CH}_{2}\right), 118.8(=\mathrm{CCl}), 124.5(\mathrm{~N}-\mathrm{C}=), 127.2(\mathrm{Ph}), 128.8$ $(\mathrm{Ph}), 129.3(\mathrm{Ph}), 135.8(\mathrm{Ph}), 166.0(\mathrm{C}-\mathrm{N})$. Found, \%: C 47.08; $\mathrm{H}$ 3.06; N 21.94. $\mathrm{C}_{10} \mathrm{H}_{8} \mathrm{Cl}_{2} \mathrm{~N}_{4}$. Calculated, \%: C 47.08; H 3.16; N 21.96.

\section{FUNDING}

The work was carried out within the framework of the State Program of Scientific Research of the Republic of Belarus "Chemical Processes, Reagents and Technologies, Bioregulators and Bioorganic Chemistry" (project 2.1.01.01).

\section{CONFLICT OF INTEREST}

No conflict of interest was declared by the authors.

\section{OPEN ACCESS}

This article is licensed under a Creative Commons Attribution 4.0 International License, which permits use, sharing, adaptation, distribution and reproduction in any medium or format, as long as you give appropriate credit to the original author(s) and the source, provide a link to the Creative Commons license, and indicate if changes were made. The images or other third party material in this article are included in the article's Creative Commons license, unless indicated otherwise in a credit line to the material. If material is not included in the article's Creative Commons license and your intended use is not permitted by statutory regulation or exceeds the permitted use, you will need to obtain permission directly from the copyright holder. To view a copy of this license, visit http://creativecommons.org/licenses/by/4.0/.

\section{REFERENCES}

1. Ostrovskii, V.A., Popova, V.A., and Trifonov, R.E., Adv. Heterocycl. Chem., 2017, vol. 123, p. 1. https://doi.org/10.1016/bs.aihch.2016.12.003

2. Kizhnyaev, V.N. and Vereshchagina, L.I., Russ. Chem. Rev., 2003, vol. 72, no. 2, p. 143. https://doi.org/10.1070/RC2003v072n02ABEH000731

3. Myznikov, L.V., Vorona, S.V., Artamonova, T.V., and Zevatskii, Yu.E., Russ. Chem. Bull., 2016, vol. 65, p. 923.

https://doi.org/10.1007/s11172-016-1394-z

4. Zeng, J., Cheng, C., Huang, B., Huang, C., and Chen, J., Sensors and Actuators (B), 2017, vol. 243, p. 234. https://doi.org/10.1016/j.snb.2016.11.148

5. Zeng, J., Cheng, C., Chang, C., Huang, C., and Chen, J., Dyes and Pigments, 2017, vol. 139, p. 300. https://doi.org/10.1016/j.dyepig.2016.11.047

6. Aleshunin, P.A. and Ostrovskii, V.A., Russ. J. Org. Chem., 2012, vol. 48, no. 6, p. 877. https://doi.org/10.1134/S1070428012060255

7. Aleshunin, P.A., Esikov, K.A., Dolgushin, F.M., and Ostrovskii, V.A., Russ. J. Org. Chem., 2012, vol. 48, no. 11, p. 1464. https://doi.org/10.1134/S1070428012110097

8. Myznikov, L.V., Melnikova, Yu.V., Baichurin, R.I., Artamonova, T.V., and Zevatskii, Yu.E., Russ. J. Gen. 
Chem., 2018, vol. 88, no. 2, p. 216.

https://doi.org/10.1134/S1070363218020068

9. Kaberdin, R.V. and Potkin, V.I., Russ. Chem. Rev., 1994, vol. 63, no. 8, p. 641.

https://doi.org/10.1070/RC1994v063n08ABEH000109

10. Dmitrieva, U.N., Ramsh, S.M., Zevatskii, Yu.E., Artamonova, T.V., and Myznikov, L.V., Chem. Heterocycl. Compd., 2011, vol. 48, no. 2, p. 355.

https://doi.org/10.1134/S1070363218020068
11. Burla, M.C., Caliandro, R., Carrozzini, B., Cascarano, G.L., Cuocci, C., Giacovazzo, C., Mallamo, M., Mazzone, A., and Polidori, G., J. Appl. Cryst., 2015, vol. 48, p. 306. https://doi.org/10.1107/S1600576715001132

12. Sheldrick, G.M., Acta Crystallogr. (C), 2015, vol. 71, p. 3.

https://doi.org/10.1107/S2053229614024218

13. Spek, A.L., Acta Crystallogr. (D), 2009, vol. 65, p. 148. https://doi.org/10.1107/S090744490804362X 